\title{
11
}

\section{Assertion, Denial, Accepting, Rejecting, Symmetry, and Paradox}

\author{
Greg Restall
}

\subsection{Introduction}

Here are my assumptions about assertion, denial, accepting, rejecting, consequence, and negation. I will not take the time to defend these assumptions here: I discuss them elsewhere (Restall 2005).

Assertion and denial are speech acts, related to the cognitive states of accepting and rejecting. Assertion typically expresses acceptance, and denial typically expresses rejection. If I assert that the cat is on the mat, then this typically expresses my accepting that the cat is on the mat. If I deny that the cat is on the mat is the case, then this typically expresses my rejecting that the cat is on the mat. These speech acts and related cognitive states are equally rationally constrained by a relation of logical consequence. If the claim that the cat is on the mat (abbreviate this claim as " $A$ ") entails the claim that some feline is on the mat (abbreviate this claim as "B", so the claim of entailment is written " $A \vdash B$ ") then accepting that the cat is on the mat and rejecting that some feline is on the mat is logically incoherent. Similarly, asserting that the cat is on the mat and denying that some feline is on the mat is likewise logically incoherent. We do not need to make any assumption about the relationship between these properties of speech acts and cognitive states: perhaps the coherence or otherwise of collections of speech acts arises out of the coherence or otherwise of the cognitive states they express. Perhaps, on the other hand, the coherence or otherwise of the cognitive states depends on the commitments incurred by engaging in the speech acts which express them. Perhaps instead, the notion of coherence, between claims, states, and perhaps other items too, is fundamental and it is only through identifying relations of coherence or incoherence that we identify what is said or what is believed. Such 
niceties are important for the general project of understanding logic, language, belief, and meaning, but we do not need to settle those issues here.

More important for us are the properties of coherence already inherent in any picture like this. The standard account of logical consequence takes the relation $\vdash$ to be reflexive and transitive. This tells us immediately that it is never coherent to simultaneously assert and deny the same claim $A$; that it is never coherent to both accept and reject $A$. This is the reflexivity of consequence: $A \vdash A$. Similarly, the transitivity of consequence tells us that if $\mathrm{A} \vdash \mathrm{B}$ and $\mathrm{B} \vdash \mathrm{C}$ then $\mathrm{A} \vdash \mathrm{C}$. Contraposing, it follows that if $A \forall \forall C$ (that is, if it is coherent to assert $A$ and deny $C)$ then it is coherent to assert $A$ and deny $B$, or coherent to assert $B$ and deny $C$. This means, after a little fiddling, that if it is coherent to assert $A$ and deny $C$ then either it is also coherent to assert $B$, along with asserting $A$ and denying $C$, or it is also coherent to deny $B$, along with asserting $A$ and denying $C .{ }^{1}$ The arbitrary $B$ is either coherently assertible, though not necessarily assertible with any warrant or evidence, or it is coherently deniable. There will never be a situation which, on the basis of logic alone, rules out asserting $B$ and also rules out denying it. One way to see this is that if logic alone rules out asserting B, then B has been ruled out, and we have every reason to deny it. This gives us very mild versions of the law of "non-contradiction" - it is incoherent both to assert and to deny $A$, or $A \vdash A-$ and of the law of "excluded middle" - it is coherent either to assert $A$ or to deny it. I say that these are very mild versions of these laws, because they are acceptable to the gap and glut theorists, who reject the law of the excluded middle and the principle of non-contradiction respectively. Some gap theorists take $A \vee \neg A$ to fail for some instances of $A$, and hence, allow for the possibility that both $A$ and $\neg A$ be rejected. That is, the friend of gaps sometimes rejects $A$ without thereby accepting $\neg A$. Symmetrically, the friend of gluts takes $A \wedge \neg A$ to succeed for some instances of $A$, and hence, allows for the possibility that both $A$ and $\neg A$ be accepted. That is, the friend of gluts sometimes accepts $\neg A$ without thereby rejecting $A$.

Noticing this symmetry between gap and glut treatments and their connection to assertion, denial, and negation is not original with me (Parsons 1984; 1990). The original contribution in this chapter is the treatment of this phenomenon in the context of multiple premise, multiple conclusion consequence, and it's to this that we turn.

The vocabulary of assertion and denial gives us a way to explain consequence not only as applying to arguments with a sole premise or a sole conclusion, but as relating premises to conclusions. If $\mathrm{A} \vdash \mathrm{B}$ tells us that asserting $\mathrm{A}$ and denying

\footnotetext{
${ }^{1}$ The fiddling involves using the "weakening" structural rule, and transitivity in its full generality. If $\mathrm{X} \vdash \mathrm{B}, \mathrm{Y}$ and $\mathrm{X}, \mathrm{B} \vdash \mathrm{Y}$ then $\mathrm{X} \vdash \mathrm{Y}$ : the so-called "cut rule."
} 
$B$ is to make an error of logic, then if $X$ and $Y$ are collections of statements, we can think of $X \vdash Y$ as telling us that asserting each element of $X$ and denying each element of $Y$ is to make an error of logic.

This generalization to multiple premise and multiple conclusion logic is not startling or original. It has been known to proof theorists at least since Gentzen's groundbreaking work in the 1930s (Gentzen 1935). The philosophical significance of multiple conclusion consequence is, however, much disputed (Shoesmith and Smiley 1978). Instead of developing the argument here, I will take it as read that multiple conclusion consequence makes sense and leave the defence of the notion to another place (Restall 2005). One way to make sense of the notion of multiple conclusion consequence is to work with it and consider its properties, and to this we will briefly turn.

Allowing sets of statements as premises and conclusions opens the way for the empty set of premises. To say that \{\}$\vdash Y$ (which we write simply as ' $\vdash Y$ ') means that the combination of asserting every member of the empty set while denying every member of $Y$ is ruled out. The empty set has no members, so we need do nothing to assert every member of the empty set. So this means simply that denying every member of $Y$ is ruled out. In the case where $Y$ is a single element set, $\{A\}$, then $\vdash\{A\}$ (which we write as ' $\vdash A$ ') tells us that $A$ is undeniableit is true on the basis of logic alone, a tautology. This, of course, trades on the move from undeniability to truth. It is important to recall here that we are not talking of what evidence or warrant may be available for a claim, but simply what position (pro or con) may be coherently taken up concerning it. If we wish to come to a position concerning $A-$ if the question of whether or not $A$ has arisen, and we wish to resolve it-then if $A$ is undeniable, the only resolution possible is to assert it.

Similarly, we may replace "assert" by "deny" and consider an empty conclusion set of the sequent $X \vdash Y$. In that case we have $X \vdash$, which tells us that asserting every member of $X$ while denying every member of the empty set is ruled out. Just as with asserting, denying every member of the empty set is trivial, as there's nothing to do: we never fail to deny each member of the empty set. So, $X \vdash$ if and only if asserting each member of $X$ is ruled out. Logic tells us that at least one member of $X$ is false. In the case where $X$ is a single element set $\{A\}$, then $A \vdash$ tells us that $A$ is false on the basis of logic alone: it is inconsistent. An empty conclusion set gives us a guide to what to deny on the basis of logic alone, given that we wish to resolve the issue of whether to assert or to deny $A$.

Finally, consider the transitivity of consequence. We would like to generalize the straightforward claim that if $A \vdash B$ and $B \vdash C$ then $A \vdash C$. In the case of more than one premise, we have the following deduction: 


$$
\frac{X \vdash B \quad X^{\prime}, B \vdash C}{X, X^{\prime} \vdash C}
$$

since the extra premises $X$ and $X^{\prime}$ simply pile up. If $X$ suffices to deduce $B$, and $X^{\prime}$ and $B$ suffice for $C$, then simply plug $X$ in for B in the proof of $C$. Similarly, if we have multiple conclusions (but single premises) we get

$$
\frac{A \vdash B, Y^{\prime} B \vdash Y}{A \vdash Y, Y^{\prime}}
$$

as we plug in the proof from $B$ to $Y$ in the conclusion of the proof from $A$ to either $B$ or $Y^{\prime}$, to conclude from $A$ the multiple conclusions $Y$ or $Y^{\prime}$. Now, in the case of multiple premises and multiple conclusions, we combine the insights:

$$
\frac{X \vdash B, Y^{\prime} \quad X^{\prime}, B \vdash Y}{X, X^{\prime} \vdash Y, Y^{\prime}}
$$

If we can infer from $X$ to either $B$ or $Y^{\prime}$, then plug in the proof from $X^{\prime}$ and $B$ to $Y$ here. To do this we gain some extra premises $X^{\prime}$, and we leave the alternate conclusions $Y^{\prime}$ to one side. If $B$ applies, though, we can (with $X^{\prime}$ ) deduce $Y$. So, if we have $X$ and $X^{\prime}$ we may deduce either $Y$ (via $X^{\prime}$ and $B$ ) or $Y^{\prime}$ (via $X$ ), as desired. This is the Cut rule, and it will be important in what follows. Let's return, now, to the consideration of the paradoxes. Priest takes symmetry between gap and glut accounts to be broken by the presence of the strengthened liar paradox.

$\langle\mathrm{sL}\rangle$ This sentence is not true.

Priest reasons as follows: the gap theorist is committed to $\langle\mathrm{SL}\rangle$ not being true, since it is a truth-value gap. But $\langle\mathrm{SL}\rangle$ itself says that it's not true, so the gap theorist contradicts herself by both saying that $\langle\mathrm{SL}\rangle$ is not true (by virtue of the analysis admitting that it has a truth-value gap) and thereby asserting $\langle\mathrm{sL}\rangle$.

But we have already seen that the gap theorist need not follow Priest in this reasoning. The gap theorist can avail herself of the distinction between asserting a negation and denying. The gap theorist can deny that $\langle\mathrm{SL}\rangle$ is true without thereby committing herself to the negation of $\langle\mathrm{SL}\rangle$. The friend of gluts cannot rule out such a move by the defender of gaps, since friends of gluts also split assertion of negations and denials, albeit in the opposite direction. If the gap account involves denying $\langle\mathrm{SL}\rangle$ without asserting its negation (or rejecting $\langle\mathrm{SL}\rangle$ without accepting its negation) the strengthened liar paradox is circumvented. This circumvention comes at a price. We have a denial (the denial of $\langle\mathrm{sL}\rangle$ itself) which cannot be expressed as an assertion of a negation. This denial must be expressed in some other way. Is this a cost for the friend of gaps? Perhaps it is: after all, it seems that 
we generally express denials when we assert negations, so breaking this nexus is a price to pay.

THE PRICE OF GAPS: It's not generally true that a denial of a statement is expressible by asserting the negation of that statement. We must deny $\langle\mathrm{SL}\rangle$ without asserting its negation.

However, we shall see that it is exactly the same kind of price which must be borne by the friend of gluts in response to exactly the same phenomenon. As a result, this cost cannot, on its own, be seen as a reason for favouring gluts over gaps.

To see this, consider the glut theorist's account of $\langle\mathrm{sL}\rangle$. The glut theorist's account is, on the face of it, straightforward. The sentence $\langle\mathrm{SL}\rangle$ is true, and also, it is not true. According to the friend of gluts, the strengthened liar paradox is a canonical case of a true contradiction. However, it does not follow that in the case of $\langle\mathrm{sL}\rangle$ (in the mouth of the friend of gluts, at least) the assertion "this sentence is not true" expresses a denial. The dialetheist does not both assert and deny $\langle\mathrm{SL}\rangle$. He asserts $\langle\mathrm{sL}\rangle$ and its negation. Just as we saw with the gap theorist, assertions of negations on the one hand, and denials on the other, diverge. Here, we have an assertion of a negation which cannot express a denial.

THE PRICE OF GLUTS: It's not generally true that a negation of a statement expresses the denial of that statement. We must assert the negation of $\langle\mathrm{SL}\rangle$ without denying it.

Symmetry is preserved: the friend of gluts, just like the friend of gaps, pays a price. It follows that if the strengthened liar paradox is a problem for the gap theorist and not for the glut theorist, then some symmetry-breaking principle must be applied. The onus is on partisans-both partisans of gaps, and partisans of glutsto elucidate such a principle. But what kind of principle could that be? Let's first consider some arguments due to Priest, in In Contradiction (Priest 2006, section 4.5-4.7), then an elaboration of the argument, and lastly an argument from a defender of gaps.

\subsection{The Aim of Assertion}

Priest seeks symmetry breaking principles in an account of the significance of assertion. He follows Dummett in taking assertion to aim at truth. An assertion succeeds when (and only when) it is true. ${ }^{2}$

\footnotetext{
2 This is, no doubt, correct under some understanding of what it is for an assertion to succeed, but it is by no means an unproblematic principle.
} 
To speak truly is to succeed in a certain activity. And in the context of asserting, anything less than success is failure. There is no question of falling into some limbo between the two. (Priest 2006, section 4.7)

This is fine, as far as it goes, but it does not go far enough to establish Priest's desired conclusion (as Parsons 1990 notes). The friend of truth-value gaps can agree that anything less than success (in this case, truth) is failure. Of course, anything that is not true is not true (that is, it fails to be true). The friend of gaps does not deny this. Rather, the friend of gaps denies that the liar sentence is true, and she denies that it is not true. Denial outstrips assertion of negation. Some untruths are denied without the friend of gaps asserting their negations.

However, I want to do more than repeat Parsons' straightforward explanation of why Priest's argument fails. I wish to add a reason of my own. If Priest's argument against the gap theorist works, then so does a parallel argument against the glut theorist.

To correctly deny is to succeed in a certain activity. And in the context of denial, only that which isn't a failure is success. There is no question of lying in some overlap between the two.

(It is a little hard to get the parallel right, because Priest conflates "speaking truly" with assertion.) Regardless, the argument goes as follows. The aim of denial is untruth. Anything not true is fit for denial, and only untruths are fit for denial. But pick a sentence, like $\langle\mathrm{sL}\rangle$, that the glut theorist takes to be both true and untrue. If the aim of denial is untruth, then this is to be denied. But wait a minute! According to the friend of gluts, $\langle\mathrm{sL}\rangle$ is true, and to be accepted. But then its denial fails because only untruths are to be denied, not truths. So, $\langle\mathrm{SL}\rangle$ falls into the overlap between what is to be denied and what is to be asserted.

Priest would agree that this is a bad argument against dialetheism. Not all negations express denials for Priest. The assertion of the negation of $\langle\mathrm{sL}\rangle$ is not (in the dialetheist's mouth) a denial. But how is it any worse than Priest's own argument against the gap theorist? Priest presupposes that the denial of $\langle\mathrm{sL}\rangle$ in the gap theorist's mouth is a negation. (Or at least, he has to, if he wants to move from the "failure" of the assertion (the denial that it is true) to the truth of its negation.)

Priest's second argument on the nature of assertion is similar to the first. $\mathrm{He}$ approvingly quotes Dummett:

A statement, so long as it is not ambiguous or vague, divides all states of affairs into just two classes. For a given state of affairs, either the statement is used in such a way that a man who asserted it but envisaged that state of affairs as a possibility would be held to have spoken misleadingly, or the assertion of the statement would not be taken 
as expressing the speaker's exclusion of that possibility. If a state of affairs of the first kind obtains, the statement is false; if all actual states of affairs are of the second kind, it is true.

Parsons notes four different problems with this argument. (1) Not all friends of gaps accept Dummett's premise. (2) The last sentence (concerning falsity) does not follow from what is said previously, since what is said up to this point does not concern falsity. (3) Priest's sense of "false" and Dummett's differ. (4) The desired conclusion (that the statement is either true or false) depends crucially on a close relative of the law of the excluded middle (that either a state of affairs of the first kind obtains or all actual states of affairs are of the second kind) that the friend of gaps is free to deny (Parsons 1990, n. 7). It seems to me that all four suggestions are well put. However, it is conceivable that Priest will find some way to repair the argument on these measures. Nonetheless, if Priest is able to present a principle to ensure that the final excluded middle succeeds (so, some grounds upon which to assert that either a state of affairs of the first kind obtains or all actual states of affairs are of the second kind) then the foe of gluts may argue for a similar principle. After all, Dummett's considerations rule against gluts just as well as they rule against gaps. The foe of gluts need simply argue for the denial of any conjunction of the form (a state of affairs of the first kind obtains, and all actual states of affairs are of the second kind). If that charge sticks, then this undercuts Priest's grounds for taking a statement to be both true and false. Priest's use of Dummett's condition against gaps relies on an inexplicit endorsement of an excluded middle. If that is legitimate, then we need some reason to see why an inexplicit rejection of contradiction is not also acceptable. In the absence of a symmetry breaker, we may conclude that these considerations relating assertion to truth and falsity lead us no further in choosing between gaps and gluts.

\subsection{LEM and LNC}

In a later paper, "What not? A defence of dialethic theory of negation" (Priest 1999), Priest attempts a different argument to the conclusion that there are truthvalue gluts but no gaps. The crux of the matter is, perhaps surprisingly, the assumption that negation is a contradictory forming operator. That is, the negation $\neg A$ of a statement $A$ is such that the following two theses, the law of the excluded middle (henceforth LEM) and the law of non-contradiction (henceforth LNC), both hold:

$$
A \vee \neg A \quad \neg(A \wedge \neg A)
$$


Priest argues that nothing that fails to satisfy either LEM or LNC counts as a negation. ${ }^{3}$ Such an operator might be contrary forming, or some other negationlike connective, but it does not count as negation. This argument, if it succeeds, is surely a symmetry breaker. Fortunately or unfortunately, the argument fails.

The first thing to note is that satisfying LNC and LEM does not suffice to isolate a single negation operator. After all, if $\neg$ satisfies LNC and LEM, then if C is some true statement so does $\neg^{\mathrm{C}}$, where we set

$$
\neg \mathrm{C} A:=\neg \mathrm{A} \wedge \mathrm{C}
$$

For it is surely the case that $A \vee(\neg A \wedge C)$ (at least, if $A \vee \neg A$, which we granted), and it is the case that $\neg(A \wedge(\neg A \wedge C)) \wedge C$ (since $\neg(A \wedge \neg A)$, which we granted), given the truth of $C$. Similarly, if $C$ is false, then $\neg C$, defined by setting

$$
\neg \mathrm{C} A:=\neg \mathrm{A} \vee \mathrm{C}
$$

also satisfies LNC and LEM, given LNC and LEM for the original connective $\neg$. But these are not negation connectives. If $C$ is merely contingently true, then while $A \vee \neg C A$ happens to be true, it could very easily be false (if $A$ and $C$ both fail). Merely contingently satisfying LNC and LEM is enough to count as being a negation connective. $^{4}$

What more could we ask for? Well, a natural requirement for Priest to demand is that the statements $A \vee \neg A$ and $\neg(A \wedge \neg A)$ not be just contingently true, but logically true. That is, we require:

$$
\vdash A \vee \neg A \quad \vdash \neg(A \wedge \neg A)
$$

That would rule out bizarre "connectives" such as $\neg^{\mathrm{C}}$ and $\neg \mathrm{C}$. But now you can see the symmetry objection. Why privilege one side of the turnstile over the other? Why is the way barred from the friend of gaps to say that we require LNC and LEM in the form:

$$
\mathrm{A} \wedge \neg \mathrm{A} \vdash \quad \neg(\mathrm{A} \vee \neg \mathrm{A}) \vdash
$$

That is, $A \wedge \neg A$ is to always be rejected-and this is an extremely natural way to read the law of non-contradiction-and $\neg(A \vee \neg A)$ is always to be rejected too. This is, to be sure, not a prominent formulation of the law of the excluded middle, but it has something to be said for it. After all, on Priest's dialethic account of negation, no statement truly excludes any other, so one might think that a reading

\footnotetext{
${ }^{3}$ And it certainly looks in this paper that there is nothing else to being a contradictory forming operator than satisfying LNC and LEM. Priest does not give any other account of what is required.

${ }^{4}$ Note that these connectives $\neg \mathrm{C}$ and $\neg \mathrm{C}$ suffice to show that satisfying LNC and LEM does not suffice for proving that $A$ is equivalent to its double negation, for $\neg^{C} \neg^{C} A$ is $\neg(\neg A \wedge C) \wedge C$, which is certainly not equivalent (in general) to $A$.
} 
of a thesis that is intended to exclude a possibility must be phrased in terms that make use of denial. What is excluded by the law of the excluded middle? The possibility that $A$ is in the "middle"-that is, that $A$ is neither true nor false. But $\neg(A \wedge \neg A)$ is one way to say that $A$ is neither true nor false. I conclude that, at the very least, this treatment of the law of the excluded middle is at least as plausible as Priest's rendering of $\vdash \neg(A \wedge \neg A)$ as a law of non-contradiction, and hence, the idea that negation is governed by the laws of excluded middle and noncontradiction is as good a justification for a glut theory of negation as it is for a gap theory of negation. We find no symmetry-breaking principles here, either.

\subsection{The "Reason Why" Objection}

One objection to gaps which does not appear to apply to gluts goes like this: ask the friend of gaps why she denies $\langle\mathrm{sL}\rangle$. The only answer she could give is something like "it's not true." But that is the one kind of answer not available to her. Reasons for assertions or for denials are propositions, and the friend of gaps does not have a reason for denying $\langle\mathrm{SL}\rangle$ the canonical reason for denying something: "it's just not true" is not available to her. This objection does not apply to the friend of gluts, because he does not deny $\langle\mathrm{SL}\rangle$.

What can the friend of gaps say? She can start by noting that reasons for denials are many different things, just lite reasons for assertions. Suppose I already have reason to accept $X$ and to reject $Y$. Then, a proof that $X, A \vdash Y$ gives us reason to reject $A$, since $A$, in the context of $X$, which we accept, entails $Y$, which we reject. This is exactly what the friend of gaps thinks of $\langle\mathrm{sL}\rangle$. So, let $\lambda$ be a strengthened liar sentence, and let $X: Y$ be a theory of truth sufficient to ensure the problematic fixed-point properties of $\lambda$. That is, the theory consists of statements $X$ to be accepted and $Y$ to be rejected. Perhaps $X$ is empty. Perhaps $Y$ is empty. So, we have

$$
X, \lambda \vdash \neg \lambda, Y \quad X, \neg \lambda \vdash \lambda, Y
$$

Then, applying the gap theorist's assumption $A, \neg A \vdash$ that there are no gluts, we can reason as follows:

$$
\frac{\lambda, \neg \lambda \vdash \quad X, \lambda \vdash \neg \lambda, Y}{X, \lambda \vdash Y}[\mathrm{CUT}]
$$

This proof is exactly symmetric to the glut theorist's proof that $\langle\mathrm{SL}\rangle$ is true. As before, $X: Y$ is whatever is needed to prove that the strengthened liar is a fixed point, and this time we apply the glut theorist's assumption $\vdash A, \neg A$ that there are no gaps. 


$$
\frac{\vdash \lambda, \neg \lambda \quad X, \lambda \vdash \neg \lambda, Y}{X \vdash \lambda, Y}[\mathrm{CUT}]
$$

The gap theorist's reason to deny has a parallel structure to the glut theorist's reason to assert. We merely exchange premises for conclusions, and no-gap principles with no-glut principles. There is no symmetry breaking here. The one and the same kind of proof (to the conclusion that $X, \lambda \vdash Y$ in the gap theorist's case, or $\mathrm{X} \vdash \lambda$, $\mathrm{Y}$, in the glut theorist's case) counts as the reason to reject (for the friend of gaps) and a reason to accept (for the friend of gluts).

The potential rejoinder, that a proof (by reductio) that $X, A \vdash Y$ should entail $X \vdash \neg A, Y$ (and hence, that we should go on to accept the negation of $A$ ), will cut no more ice with the gap theorist than the parallel rejoinder to be made to a glut theorist that the proof of $X \vdash A, Y$ should entail $X, \neg A \vdash Y$, and hence, to the rejection of $\neg A$. Symmetry is preserved here too.

\subsection{Sentences and Propositions}

Here is a reason to favour gaps over gluts. The liar sentence is not true because it does not express a proposition. Its negation is also not true, because that sentence does not express a proposition either. So neither $\langle\mathrm{SL}\rangle$ nor its negation is true (we deny both). This reason seems to favour gaps over gluts. Doesn't this break symmetry?

Certainly, symmetry is broken if we leave things at this point. (This is not to say that the foe of gaps and friend of gluts would be moved by this reasoning. The argument to the conclusion that $\langle\mathrm{SL}\rangle$ does not express a proposition will use principles rejected by the friend of gluts: the reasoning crucially passes to the conclusion that if $\langle\mathrm{SL}\rangle$ were to express a proposition, it and its negation would be true, but this is rejected ...) However, once we take it that seemingly syntactically well-formed sentences (such as $\langle\mathrm{SL}\rangle$ ) do not express propositions, then presumably, a parallel case is to be found with sentences that don't fail in virtue of expressing too few propositions, but rather, succeed all too well in expressing too many. If we have a sentence that expresses two propositions, then when is an assertion of that sentence acceptable? There seem to be two different choices. Either it is true when either of the propositions expressed is true (the existential reasoning), or it is true when both of the propositions expressed are true (the universal reading). In the existential case, then both the assertion of that sentence and the assertion of its negation may be acceptable. In the universal, we may have a gap. What reason might we have to choose between these two options? Do we wish to encourage assertion by allowing it to be acceptable more often, or do we wish to restrict it by imposing the tougher condition that both propositions 
expressed be true? The first option brings gluts and the second brings gaps. The mere fact of multiple expression gives us no reason to choose between them.

But if this is the case, it seems to be an option even in the case where a proposition has not been expressed. The friend of gluts could say that the assertion of a sentence expressing no proposition could, after all, be acceptable, by analogy with the universal reading in the case of multiple propositions. After all, in the empty case, every proposition expressed by the sentence (which expresses no proposition!) is true. An assertion of this sentence fails, to be sure: it fails to rule anything out. Therefore (on the universal reading, at least) the assertion is acceptable. It seems that even here, the friend of gluts has some room to manoeuvre.

My tentative conclusion, to the effect that gap and glut theories are on a par, is not popular. Almost all defenders of dialethic accounts of the paradoxes take their account to be superior to gap accounts because of $\langle\mathrm{SL}\rangle$. Almost everyone else takes truth-value gluts to be crazy, and truth-value gaps to be sane. Symmetry is a minority position. ${ }^{5}$ Furthermore, a symmetricalist can take one of two forms. Gap and glut theories could be equally good (so, we could endorse a form of Parson's agnostaletheism, an agnosticism between gaps and gluts, or perhaps a more liberal semantic theory which allows for both gaps and gluts) or they are equally $b a d$.

\section{References}

Brandom, Robert B. 2000. Articulating Reasons: An Introduction to Inferentialism. Cambridge, MA: Harvard University Press.

Dunn, J. Michael. 2000. "Partiality and its dual." Studia Logica 65:5-40.

Dunn, J. Michael and Hardegree, Gary M. 2001. Algebraic Methods in Philosophical Logic. Oxford: Oxford University Press.

Gentzen, Gerhard. 1935. "Untersuchungen über das logische Schliessen." Mathematische Zeitschrift 39:175-210, 405-31. English translation 'Investigations concerning logical deduction' in Szabo (1969, 68-131).

Parsons, Terence. 1984. "Assertion, denial, and the liar paradox." Journal of Philosophical Logic 13:137-52.

Parsons, Terence. 1990. “True contradictions." Canadian Journal of Philosophy 20:335-54.

Priest, Graham. 1999. "What not? A defence of dialetheic theory of negation." In D. Gabbay and H. Wansing (eds.), What is Negation? 101-20. Dordrecht: Kluwer.

Priest, Graham. 2006. In Contradiction. Oxford: Oxford University Press, 2nd edition.

\footnotetext{
${ }^{5}$ But friends of symmetry are in very good company. See, for example, Dunn's work on negation (Dunn 2000), and (with Hardegree) on multiple-premise, multiple-conclusion consequence as an appropriate general setting to characterize properties of different 'logics' (Dunn and Hardegree 2001, especially section 6.8).
} 
Restall, Greg. 2005. “Multiple conclusions.” In P. Hajek, L. Valdez-Villanueva, and D. Westerstahl (eds.), Proceedings of the Twelfth International Congress on Logic, Methodology, and Philosophy of Science, 189-205. London: King's College Publications.

Shoesmith, D.J. and Smiley, T.J. 1978. Multiple-Conclusion Logic. Cambridge: Cambridge University Press.

Szabo, M.E. (ed.). 1969. The Collected Papers of Gerhard Gentzen. Amsterdam and London: North-Holland. 


\section{University Library}

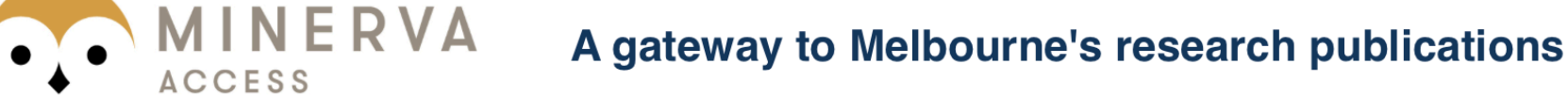

Minerva Access is the Institutional Repository of The University of Melbourne

Author/s:

RESTALL, $G$

Title:

Assertion, Denial, Accepting, Rejecting, Symmetry and Paradox

Date:

2015

Citation:

RESTALL, G. (2015). Assertion, Denial, Accepting, Rejecting, Symmetry and Paradox.

Caret, C (Ed.). Hjortland, O (Ed.). Foundations of Logical Consequence, Foundations of Logical Consequence, (1), pp.310-321. Oxford University Press.

Persistent Link:

http://hdl.handle.net/11343/58839 